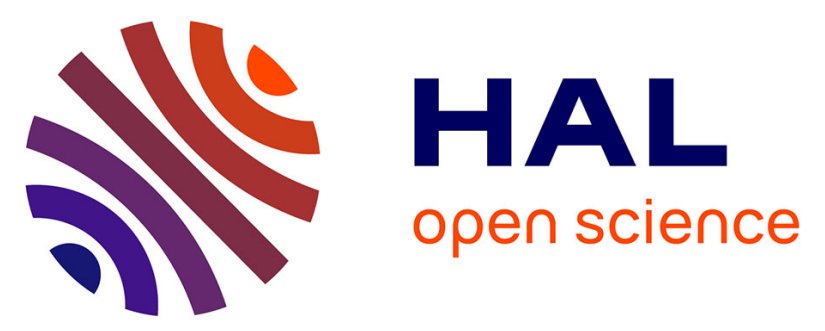

\title{
Jovian Auroral Radio Sources Detected In Situ by Juno/Waves: Comparisons With Model Auroral Ovals and Simultaneous HST FUV Images
}

C. Louis, R. Prangé, L. Lamy, P. Zarka, M. Imai, W. Kurth, J. Connerney

\section{- To cite this version:}

C. Louis, R. Prangé, L. Lamy, P. Zarka, M. Imai, et al.. Jovian Auroral Radio Sources Detected In Situ by Juno/Waves: Comparisons With Model Auroral Ovals and Simultaneous HST FUV Images. Geophysical Research Letters, 2019, 46 (21), pp.11606-11614. 10.1029/2019GL084799 . hal-03127354

\section{HAL Id: hal-03127354 \\ https://hal.science/hal-03127354}

Submitted on 29 Nov 2021

HAL is a multi-disciplinary open access archive for the deposit and dissemination of scientific research documents, whether they are published or not. The documents may come from teaching and research institutions in France or abroad, or from public or private research centers.
L'archive ouverte pluridisciplinaire HAL, est destinée au dépôt et à la diffusion de documents scientifiques de niveau recherche, publiés ou non, émanant des établissements d'enseignement et de recherche français ou étrangers, des laboratoires publics ou privés.

$$
\text { Copyright }
$$




\section{Geophysical Research Letters}

\author{
RESEARCH LETTER \\ 10.1029/2019GL084799 \\ Key Points: \\ - The position of the sources of the \\ broadband Kilometric, Hectomet- \\ ric and Decametric auroral radio \\ emissions is statistically determined \\ from in situ source crossings by \\ Juno/Waves \\ - All radio sources are found to be \\ located on the same set of magnetic \\ field lines, with $\mathrm{M}$ ranging from 10 \\ to 60 , and are connected to the main \\ UV oval \\ - Correspondence between auroral \\ radio and ultraviolet emissions val- \\ idated by simultaneous auroral \\ observations by Juno/Waves (radio) \\ and HST/STIS (FarUltraviolet)
}

Correspondence to:

C. K. Louis,

corentin.louis@irap.omp.eu

\section{Citation:}

Louis, C. K., Prangé, R., Lamy, L., Zarka, P., Imai, M., Kurth, W. S., \& Connerney, J. E. P. (2019). Jovian auroral radio sources detected in situ by Juno/Waves: Comparisons with model auroral ovals and simultaneous HST FUV images. Geophysical Research Letters, 46, 11,606-11,614. https://doi.org/ 10.1029/2019GL084799

Received 31 JUL 2019 Accepted 2 OCT 2019 Accepted article online 17 OCT 2019 Published online 3 NOV 2019

(C)2019. American Geophysical Union. All Rights Reserved.

\section{Jovian Auroral Radio Sources Detected In Situ by Juno/Waves: Comparisons With Model Auroral Ovals and Simultaneous HST FUV Images}

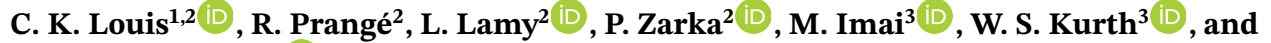 \\ J. E. P. Connerney ${ }^{4}$ iD
}

${ }^{1}$ Institut de Recherche en Astrophysique et Planétologie (IRAP), Université de Toulouse, CNRS, CNES, UPS, ,Toulouse, France, ${ }^{2}$ LESIA, Observatoire de Paris, PSL Research University, CNRS, Sorbonne Universités, UPMC Université Paris 06, Université Paris Diderot, Sorbonne Paris Cité, ,Meudon, France, ${ }^{3}$ Department of Physics and Astronomy, University of Iowa, Iowa City, IA, USA, ${ }^{4}$ NASA Goddard Space Flight Center, Greenbelt, MD, USA

\section{Introduction}

The Jovian nonthermal auroral radio spectrum is commonly split into several frequency components (Zarka, 1998; Zarka et al., 2004), including the broadband kilometric (bKOM, [10-400] kHz), hectometric (HOM, $\sim[250-3000] \mathrm{kHz}$ ), and decametric (DAM, [3-40] MHz). These boundaries are not perfectly delimited, and some emissions slightly exceed on an other range.

Similar to the auroral kilometric radiation at Earth and Saturn, the mechanism for producing the Jovian auroral radio emissions is the Cyclotron Maser Instability (CMI; Wu, 1985). This instability requires two conditions: (i) a magnetized plasma, where the electron plasma frequency $\mathrm{f}_{\text {pe }}$ is much lower than the electron cyclotron frequency $\mathrm{f}_{\mathrm{ce}}=e B / m_{e}$ (Hilgers, 1992; Zarka et al., 2001), with $B$ the magnetic field magnitude, and $e$ and $m_{e}$ the electron charge and mass; (ii) a weakly relativistic electrons previously accelerated along high-latitude magnetic field lines (MFLs) at typical energies of a few kiloelectron volts (Zarka, 1998, 2004). This leads to emission at a frequency very close to $f_{\text {ce }}$.

Ladreiter et al. (1994) used Ulysses/URAP direction-finding (DF) measures to show that the bKOM sources lie along dipolar MFLs of $\mathrm{L}=9-15$ (measured in Jovian equatorial radii $R_{J}=71,492 \mathrm{~km}$ ). More recently Imai et al. (2017) used one Juno/Waves DF measures to show that the $M$ values (similar to $L$ but for a nondipolar magnetic field model) range from 50 to 60, while Imai et al. (2019) used in situ and DF measures over six bKOM events to estimate that the $M$ ranges are [10-12] and [20-61]. 
Using Ulysses/URAP DF measures to study remotely the HOM sources, Reiner et al., (1993, 1993) showed that these sources are located on MFL of $L<6$, while Ladreiter et al. (1994) showed that the $L$ values range from 7 to 11 .

Genova et al. (1987) used Nançay and Voyager/PRA observations to study correlation between HOM and nonsatellites DAM events. They showed that the observed events originate from the same MFL, at different altitude.

These findings suggested that the various radio emissions may have different origins in different regions of the magnetosphere. Because the CMI mechanism generates emissions at or near $f_{\text {ce }}$, the three, rather arbitrarily differentiated wavelength ranges are almost certainly generated at different radial distances where the local magnetic field sets the frequency. The question to be settled is whether these sources are on a common set of field lines and only separated by source distance along the field or are they on different sets of MFL, altogether.

At the footprints of auroral MFL, bright far ultraviolet (FUV) auroral emissions are generated by collisions of downward energetic electron beams (from a few tens to a few hundreds of kiloelectron volts; Grodent, 2015; Pallier \& Prangé, 2004; Prangé et al., 1998) with Jupiter's upper neutral atmosphere. Past studies have tentatively compared radio emissions and FUV aurorae without clear conclusions. Prangé et al. (1993) led a pioneering comparative study on the power of FUV and DAM radio auroral emissions and showed a similar longitudinal variability. Gurnett et al. (2002) observed a simultaneous temporal increase in the intensity of the FUV and the HOM radio emissions. More recently, using Juno/Waves in situ and DF measures over three perijoves, Imai et al. (2019) showed six examples of partial correlation between FUV aurorae and bKOM radio emissions.

The challenge is to know if, like for the Earth (Huff et al., 1988; Menietti et al., 2011) or for Saturn (Clarke et al., 2009; Kurth et al., 2005; Lamy et al., 2009, 2013, 2018), the electron populations responsible for radio and FUV emissions are located on the same MFL or if there may be differences, as suggested by the IR/radio anticorrelation of the footprint of Io (Connerney et al., 1993).

In order to address these questions, we visually surveyed the Juno/Waves data (section 2.1) at closest approach, or perijove (PJ), from 27 August 2016 to 7 September 2018, to identify the auroral radio sources from in situ Juno measurements (section 2.2). The Hubble Space Telescope (HST) data set taken during these perijoves were selected for images strictly simultaneous with radio source crossings (section 2.3). Section 3 focuses on the statistical distribution of the various radio sources. Section 4 presents the comparison between FUV and radio data, and finally, section 5 summarizes and discusses the results.

\section{Observations and Analysis}

\subsection{Juno/Waves Data Set}

One of the main objectives of the Juno mission is to study the auroral processes (Bagenal et al., 2017), with 53-days-long polar orbits. This allows Juno to sample the auroral regions above each magnetic pole. The altitude range (from $\simeq 15 R_{\mathrm{J}}$ down to $<1 R_{\mathrm{J}}$ above the one bar level) probed in situ in these regions includes the sources of all the Jovian auroral radio components (bKOM/HOM/DAM).

The Juno spacecraft carries the Waves instrument (Kurth et al., 2017). It consists of one dipole electric antenna with a length of $4.8 \mathrm{~m}$ connected to two receivers covering the entire frequency range of Jovian radio auroral emissions. The low-frequency receiver samples two bands for the analysis of plasma and radio waves: [50 Hz to $20 \mathrm{kHz}$ ] and [10-150] kHz. The high-frequency receiver (HFR) also samples two bands: [150 kHz to $3 \mathrm{MHz}$ ] and [3-41] MHz. Juno/Waves operates with two main instrumental modes: the routine (low-resolution, survey mode) and the waveform mode (high-resolution, burst mode), which both sweep the frequency range every $1 \mathrm{~s}$ during the perijoves.

\subsection{Identification of Source Crossings}

Juno/Waves data from Perijoves 1 to 15 were visually inspected to identify radio sources encountered along the probe trajectory (Kurth et al., 2017). For the sake of simplicity, we used a single selection criterion, namely, emissions observed at frequencies lower than $1.01 \times f_{\text {ce }}$, based on Louarn et al. (2017), who measured CMI-driven radio emissions at a frequency up to $1 \%$ above $f_{\text {ce }}$. This criterion was robust enough to include both radio sources effectively crossed by Juno or very nearby ones, the uncertainty on the spatial locus of 

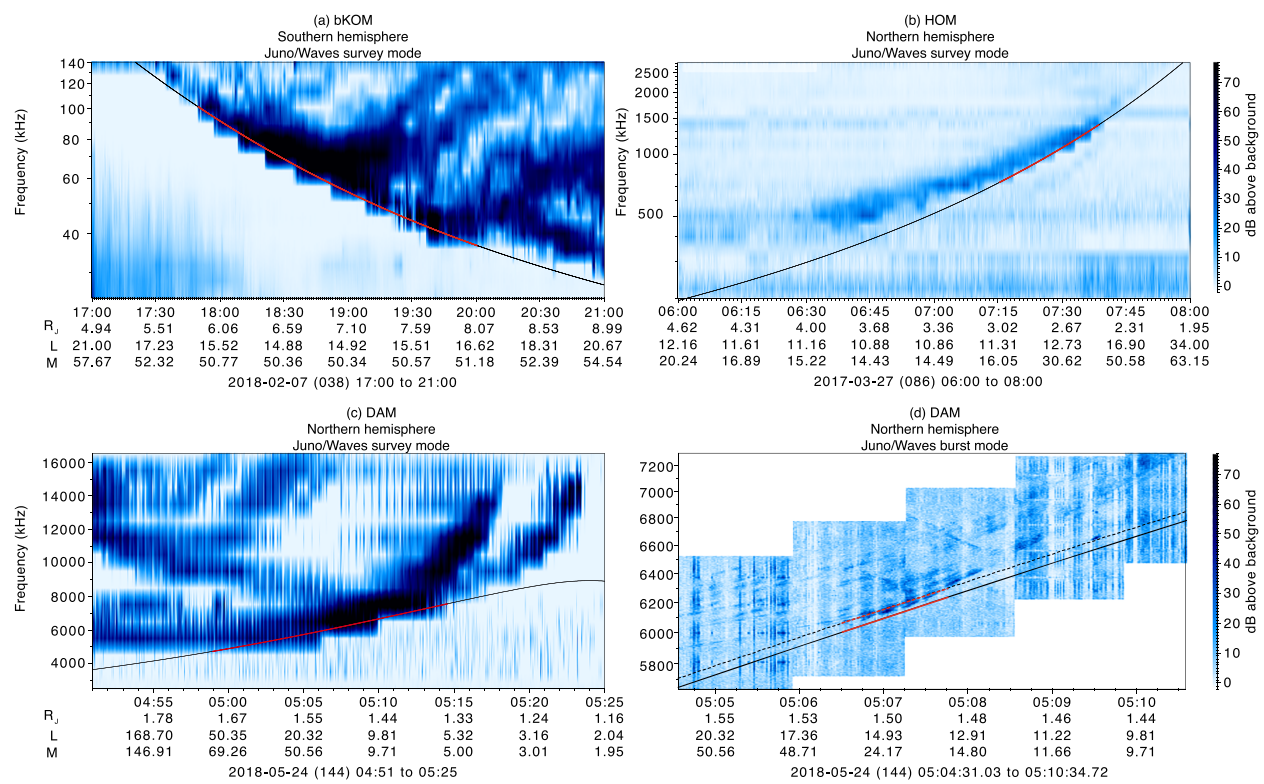

Figure 1. Panels (a) and (b) show, respectively, an example of a bKOM and a HOM source crossing (red line), and panels (c) and (d) of the same DAM source crossing. Panels (a)-(c) show Juno/Waves survey mode data, whereas panel (d) shows burst mode data. The black line indicates the local $f_{\text {ce }}$, derived from in situ Juno/MAG (Connerney et al., 2017) measurements of the magnetic field amplitude. The dashed line visible in panel (d) indicates the $f_{\text {ce }} \times 1.01$.

the latter being considered as fully negligible for the localization of the sources. For this reason, we hereafter refer to all the radio source candidates fulfilling our criterion as "local" radio sources.

Figures 1a-1c, respectively, present crossings (red line) of bKOM, HOM, and DAM sources using the survey mode. Figure 1d shows the same DAM source crossing as in Figure 1c, but displayed with the burst mode. In this high-resolution dynamic spectrum, we clearly see some modulations every $15 \mathrm{~s}$, due to the rotation of the probe and of the dipole antenna orientation.

The study of the bKOM and HOM source crossings was carried out using the survey mode, whereas that of the DAM source crossings was made with the burst mode. Indeed, for the same time duration (e.g., $20 \mathrm{~min}$ ) the spectral range is much larger in the DAM case (Figure $1 \mathrm{c}, 4.5 \times 10^{3}$ to $7.5 \times 10^{3} \mathrm{kHz}$ ) than in the HOM's or the bKOM's one (Figure 1a, 102 to $36 \mathrm{kHz}$ ). Therefore, the spectral resolution of the survey mode is no longer sufficient and its use induces a large spatial uncertainty on the location of these sources.

\subsection{HST/STIS Data Set}

HST observations of Jupiter's FUV aurorae were acquired during Juno pole-to-pole flybys from PJ\#3 to PJ\#7 (Grodent et al., 2018). They have been made public through the Mikulski Archive for Space Telescopes Space Telescope Science Institute archive, and we accessed them through the public APIS service (Lamy et al., 2015). The images were obtained with the FUV Multi-Anode Microchannel Array detector using the Strontium Fluoride filter F25SrF2 (148-nm central wavelength, $28 \mathrm{~nm}$ full width at half maximum), which tracks auroral emission from $\mathrm{H} 2$ bands. Each exposure covered the available observing time in a given HST orbit $(2,484 \mathrm{~s})$. In this study, we selected the images of the Space Telescope Imaging Spectrograph during which Juno passed within the auroral radio sources. The data, acquired with the time-tag mode, provide the arrival time of photons recorded on the Multi-Anode Microchannel Array detector at a $125 \mu$ s time resolution and thus enabled us to reconstruct shorter subexposures over relevant time intervals strictly simultaneously with each radio source crossing, once corrected from the light travel time between Juno and the Earth.

Each image was visually fitted to a planetary disc to maximize the telescope pointing accuracy. A disc background subtraction was then applied using the first-order Minnaert function (Vincent et al., 2000) fitted to the disc up to midlatitudes and extrapolated to high latitudes. The background-subtracted images were then converted into unabsorbed total brightness over the $\mathrm{H} 2$ bands and projected onto the polar atmosphere at 300 km (Gustin et al., 2016; Prangé et al., 1998). 


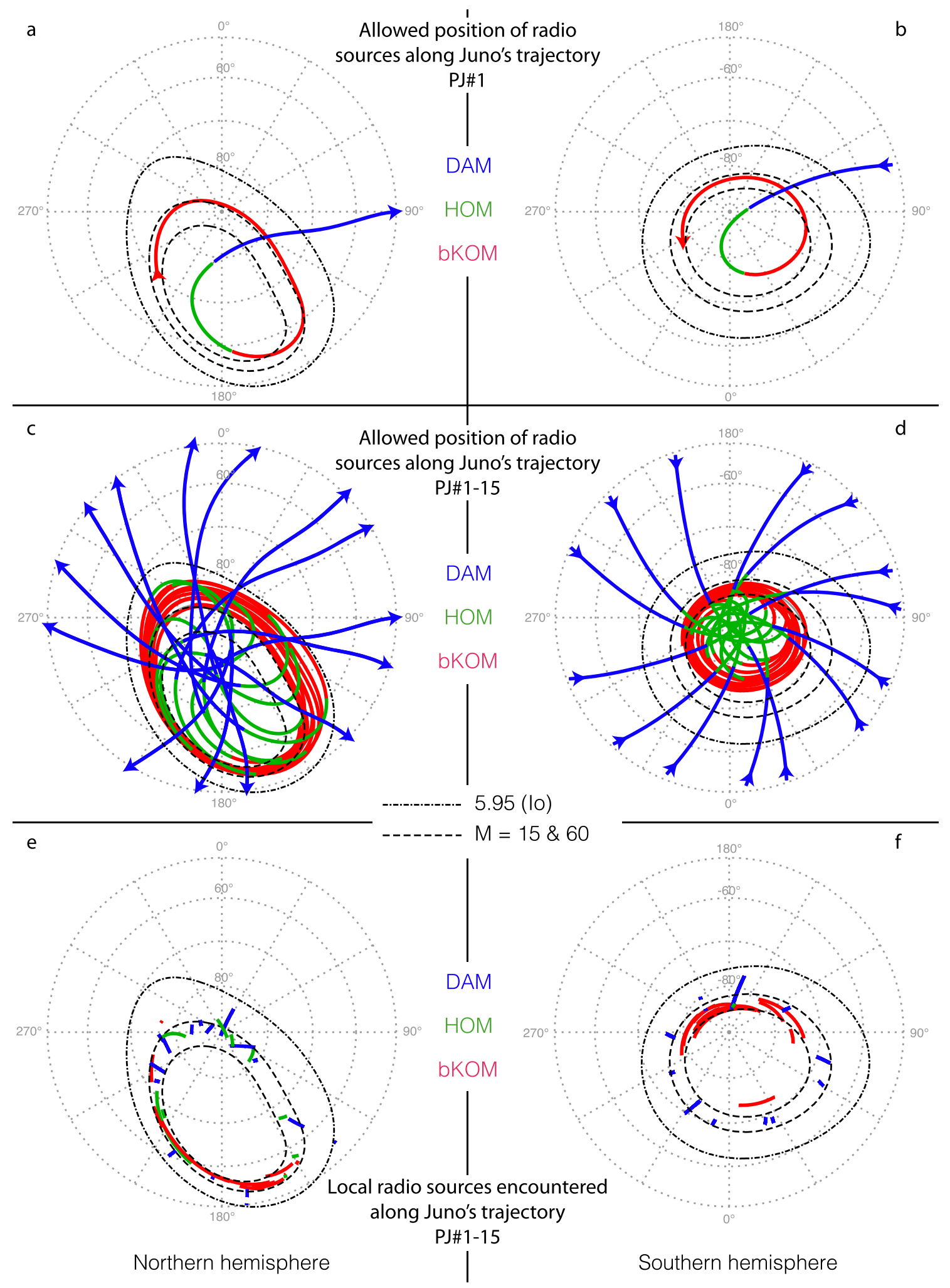

Figure 2. Planetocentric projection at $300 \mathrm{~km}$ above the 1-bar level of Juno's magnetic footprint, for the northern (panels a, c, and e) and southern hemispheres (panel b, d, and f), using the JRM09 magnetic field model (Connerney et al., 2018) and the Connerney et al. (1981) current sheet model. Panels (a) and (b) display in red, green, and blue the allowed position of bKOM/HOM/DAM radio sources along Juno's trajectory for PJ\#1, while panels (c) and (d) display it for PJ\#1-15 (except PJ\#2). Panels (e) and (f) only display the local radio sources effectively encountered (in color). In all panels, the black dashed lines indicate the magnetic footprint of the field lines whose $M$ is 15 and 60 . The black dash-dotted line indicates the Io's magnetic footprint. 


\begin{tabular}{lccc}
$\begin{array}{l}\text { Table 1 } \\
\text { Characteristics of }\end{array}$ & Auroral & Radio Source Crossings \\
\hline $\begin{array}{l}\text { Auroral radio } \\
\text { components }\end{array}$ & $\begin{array}{c}\text { Number of } \\
\text { crossed sources }\end{array}$ & $\begin{array}{c}\text { Total duration } \\
\text { (min) }\end{array}$ & $\begin{array}{c}\text { M shell } \\
\text { apex }\end{array}$ \\
\hline bKOM north & 15 & 421 & $10-62$ \\
bKOM south & 11 & 972 & $20-66$ \\
HOM north & 9 & 160 & $12-58$ \\
HOM south & 1 & 3 & $48-58$ \\
DAM north & 14 & 30.83 & $7-61$ \\
DAM south & 12 & 33.42 & $7-60$ \\
Io-DAM north & 2 & 0.8 & $4-6$ \\
Total & 64 & $1,621.05$ & \\
\hline
\end{tabular}

\section{Mean Spatial Distribution of the Jovian Radio Sources}

Figures $2 \mathrm{a}$ and $2 \mathrm{~b}$ display northern and southern polar projections of Juno's trajectory magnetic footprint drawn for a period of $10 \mathrm{hr}$ both sides of PJ\#1. The segments along the path where the magnetic field magnitude at Juno allows emission at either bKOM, HOM, and DAM $f_{\text {ce }}$ frequencies are displayed in red, green, and blue, respectively. The Juno's eccentric polar orbit brings it to arrive gradually above the north pole at high altitude (Figure 2a) where it spends a few hours with the polar/auroral zone rotating below (hence winding and long projections at high latitude). During this time interval, Juno is at altitudes where the magnetic field corresponds to the bKOM frequency range (in red). The probe then gradually decreases its altitude, at increasing speed, above the northern low-latitude auroral region toward its equatorial perijove (hence very short projections quasi-normal to the ovals), where the magnetic field encountered corresponds to the HOM (in green) and then the DAM frequencies (in blue). The opposite happens (Figure 2b) in the southern hemisphere (SH). Figures $2 \mathrm{c}$ and $2 \mathrm{~d}$ are similar to Figures $2 \mathrm{a}$ and $2 \mathrm{~b}$, but for PJ\#1-15 (without PJ\#2 where the instruments were offline). It thus provides a reasonable statistical coverage of the regions where each type of radio emissions could possibly be emitted. Figures $2 \mathrm{e}$ and $2 \mathrm{f}$ display the location where such local sources were effectively detected. It reveals that for all three types or radio emissions, the 64 detected sources, at the exception of two DAM sources projected in the northern hemisphere $(\mathrm{NH})$ near the Io footprint model oval at $\sim 130^{\circ}$ and $\sim 170^{\circ}$ of longitude, lie along MFL approximately confined between the reference auroral ovals of $M=15$ and 60 . The other two sources are connected with the magnetic footprint of Io's orbit downstream of Io and they are attributed to DAM emission induced by Io's wake (Szalay et al., 2018).

Table 1 summarizes the results of the number of crossed sources, the total time spent in the sources, and the $M$ ranges, for each component in each hemisphere.

The bKOM sources encountered by Juno (in red) are located on MFL of $M=$ [14-62] (and one case at $M=10)$ in the NH (15 cases distributed over $8 \mathrm{PJ})$ and $M=[20-66]$ in the $\mathrm{SH}$ (11 cases over $8 \mathrm{PJ})$. For the HOM (in green), the range is $M=[12-58]$ in the $\mathrm{NH}$ ( 9 cases over $9 \mathrm{PJ}$ ), and $M=[48-58]$ in the $\mathrm{SH}$ ( 1 case over $1 \mathrm{PJ}$ ). Regarding the DAM auroral radio component, it lies in the range $M=[7-61]$ in the NH (14 cases over $14 \mathrm{PJ}$ ), and $M=$ [7-60] in the SH (12 cases over $11 \mathrm{PJ})$. This in situ analysis of local radio sources thus establishes that the three components of the auroral radio emissions, bKOM, HOM, and DAM, come from the same range of MFL, contrary to some earlier results. The range of active field lines encountered here extends from $M=7$ to 66 . These typically define auroral field lines connected to the main and "secondary" (at lower latitudes) FUV auroral ovals. We will further discuss these results in section 5.

\section{Comparison of Radio Sources With FUV Observations}

The analysis of the entire HST observation set of Jupiter acquired close in time with Juno's perijove revealed that only five FUV images were acquired simultaneously with Juno radio source crossings. A sixth crossing occurred just 2.5 min before the beginning of an HST exposure. Out of these, two FUV images were simultaneous with the traversal of bKOM sources (southern radio sources crossed on 27 March 2017 between 12:10 hr and 13:10 hr and on 19 May 2017 between 09:50 hr and 10:20 hr). These have already been discussed by Imai et al. (2019) using Juno/ultraviolet spectrograph data (UVS, Gladstone et al., 2017). Our findings are in agreement with their results, so they need not be presented here. 


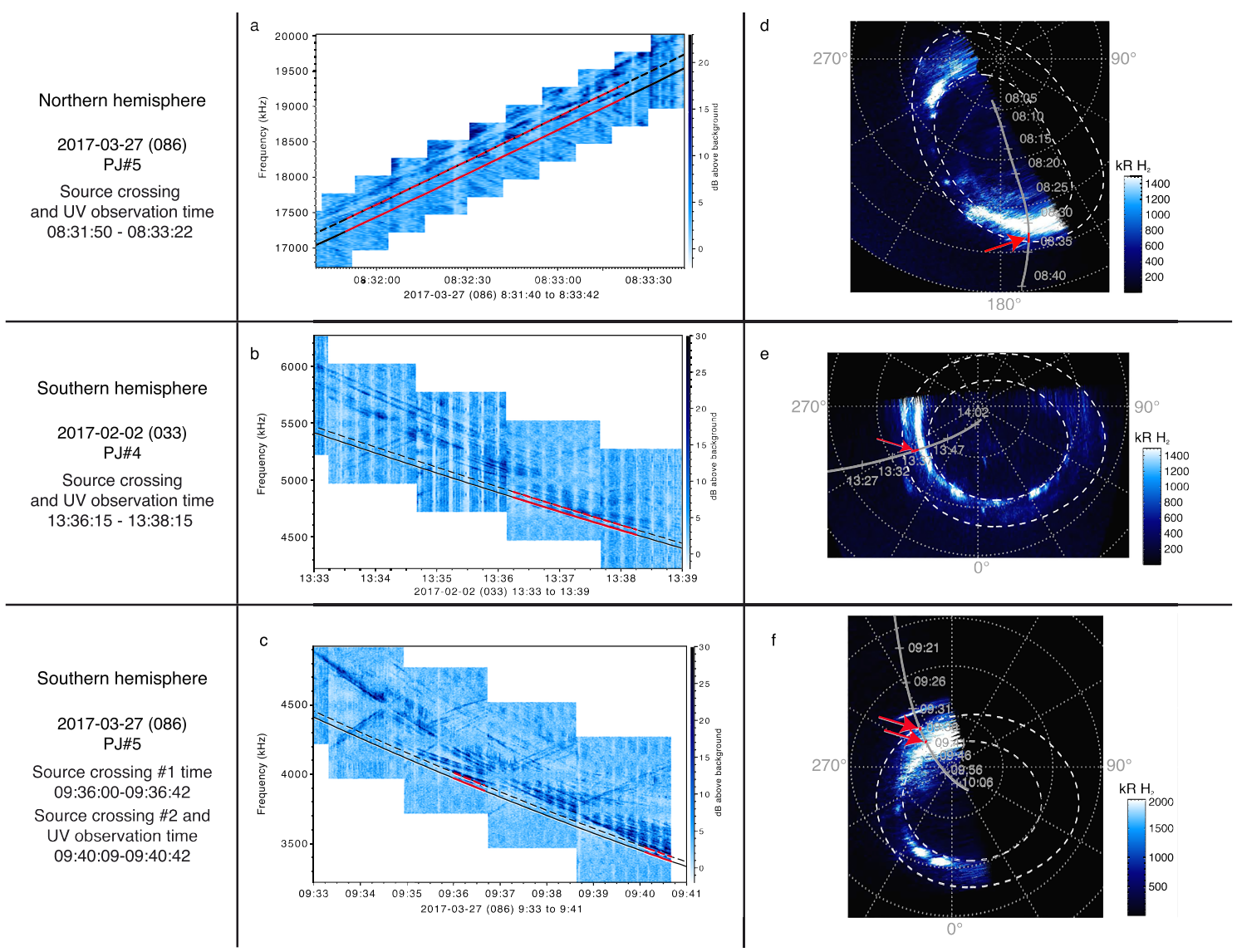

Figure 3. Panels (a)-(c) show DAM source crossing (red line) in the same way as Figure 1 . The black line indicates the local $f_{\text {ce }}$ and the black dashed line $f_{\text {ce }} \times 1.01$. Panels (d)-(f) show the polar projection—at $300 \mathrm{~km}$ in planetocentric coordinates and computed with the JRM09 magnetic field model-of Juno's path (gray solid line) with the DAM radio source crossing overplotted in red (corresponding to the one plotted on the left). They are superimposed on the polar projection of far ultraviolet (UV) data emissions recorded by Hubble Space Telescope/Space Telescope Imaging Spectrograph simultaneously with the source crossings (corrected for the light travel time). The white dashed lines indicate the model auroral ovals at the footprint of the magnetic field line with $M=10$ and 60. The time intervals are indicated in Juno time for each observations.

In this study we focus on the other four cases that all involve DAM radio source crossings, displayed Figures 3a-3c. Note that some signal almost perpendicular to $f_{\text {ce }}$ (black solid line) are visible here, drifting to the upper frequencies (e.g., panel c, between 9:33 and 9:34.5 hr and 4200-4500 kHz). These signals are artifacts, caused by the HFR (reflections of the real signals).

Figures $3 \mathrm{~d}-3 \mathrm{f}$ show the superposition of Juno's magnetic footprint (in gray solid line) with the radio source crossings intervals indicated in red, and of the polar projections of the FUV aurorae radiated during the same exact time interval. Panels (a) and (d) show a case that occurred in the NH on 27 March 2017, panels (b) and (e) a case in the SH on 2 February 2017, and panels (c) and (f) two cases on the SH on 27 March 2017. For Panel (f), the first crossing is detected 2.5 min before the start of the long HST exposure covering the second crossing; thus, we used the same simultaneous FUV subimage once we had checked that the aurora was stable (in brightness and shape).

This figure reveals that each of the four DAM radio source crossings is located on MFL connected to the equatorial edge of the FUV auroral oval (on a structure or a sub-structure). However, the vast majority of Juno's path magnetically connected to FUV auroral emissions does not host any radio source. This could indicate that the presence of an aurorally active MFL is a necessary condition to trigger radio emissions but that it is not sufficient and that other physical plasma conditions are needed for their generation. We will come back to this in section 5 . 


\section{Summary and Discussion}

All the earlier results on the location of Jovian radio sources suggested that bKOM, HOM, and DAM sources were radiated from MFL connected to different equatorial distances. But they had been derived from a limited sample of only remote measurements (the anisotropy of the emission can mislead the detection geometry) and less accurate magnetic field models (except for ; Imai et al., 2019), introducing a bias in the apex of the MFL hosting the sources.

In this study we have performed in situ detections of the sources along Juno's path, which avoid biases on the observations geometry. In addition, the analysis of the first 15 perijoves has provided an extended coverage of polar/aurora region, even though some regions were less accurately sampled (i.e., the $M$ apexes have all been sampled except $M<20$ for the bKOM in the SH). We could identify 64 local radio sources, and although this sample is still statistically modest, we have established that non-Io-DAM, HOM, and bKOM are encountered on the same family of MFL, with the bulk of the sources on M shells ranging from 15 to 60 (see Figures $2 \mathrm{e}$ and $2 \mathrm{f}$ and Table 1), which statistically correspond to the average extent of the main auroral oval, that is, mapping to the region of corotation breakdown (Cowley et al., 2002; Grodent, 2015; Grodent et al., 2018; Nichols \& Cowley, 2004). Few cases extend, or are located, (i) on outer MFL (one case in the NH and four cases in the southern one for the bKOM) and (ii) on inner MFL (five cases for the DAM in both hemisphere, two cases for the HOM, and the bKOM in the NH). These range of $M$ shells also correspond to auroral zones (secondary ovals, plasma injections signatures, ... ). The findings for this last MFL range seem to depend somewhat on the frequency ranges, with the DAM sources hosted by more inner MFL ( $M$ down to 7 in both hemispheres) than the HOM ( $M$ down to 12 in the NH) and the bKOM ( $M$ down to 14 in the $\mathrm{NH}$, except one case at $M=10$ ). However, this observation is based on few cases, and one should wait until the end of the mission for a geometrically complete and statistically significant coverage of the auroral radio emissions before validating this suggestion.

The longitude coverage is best for the DAM (Figures 2c and 2d), since Juno crosses MFL of apex $M=$ [10-60] each time at a different longitude, where sources are encountered almost each time (Figures 2e and 2f). By contrast, for the bKOM, we do not encounter sources at all longitudes, but this is a consequence of Juno's path (Figures 2c and 2d). However, we must highlight that the probe crossed the aurorally "active" MFL of apex $15-60$ only for longitudes between $150^{\circ}$ and $250^{\circ}$ in the $\mathrm{NH}$ and $90^{\circ}$ and $300^{\circ}$ in the $\mathrm{SH}$, so that the apparent longitude dependence of the source crossings is not real. In these longitude ranges, we detect sources most of the time (Figures 2e and 2f).

Concerning the HOM, the longitudes in the NH are well covered since Juno crosses the MFL of apex $M=$ [10-60] at different longitudes for each orbit. On the other hand, it only crosses four times these MFL in the SH. Furthermore, the interpretation is made harder due to both the lower sensitivity of the HFR-low-frequency band ([150 kHz to $3 \mathrm{MHz}])$, and the presence of a variable interference in the $[\simeq 120$ - to $700]-\mathrm{kHz}$ frequency range, which affected most of the perijoves. Nevertheless, in the $\mathrm{NH}$, the probe detected nine sources (distributed over $9 \mathrm{PJ}$ ), where it spent a total of $160 \mathrm{~min}$. The sources were also located on the same range of MFL than bKOM and DAM, with $M=[12-58]$.

Finally, the last section of this article compared the FUV aurorae and radio data. Previous studies suggested a link between DAM and FUV (Prangé et al., 1996), HOM and FUV (Gurnett et al., 2002), and bKOM and FUV (Imai et al., 2019). In our study, we have investigated six cases of simultaneous radio and FUV observations. They all show that FUV emissions are observed at the footprint of MFL carrying DAM and bKOM radio sources. However, the observed radio emissions do not match the strongest FUV emissions, and the vast majority of MFL connected to FUV auroral emissions—a permanent and extended phenomenon-does not host radio sources-localized and transient. By contrasts all four crossings occurred on the equatorial edge of auroral structures, which if confirmed by further observations, could bring some clues to underlying plasma process. Like at Earth and Saturn, the electron populations responsible of auroral radio emissions are located on the same MFL that the ones responsible to FUV emissions but, as at Saturn (Lamy et al., 2018), the correspondence seems to be only partial. This could imply, among other conditions, that (i) the acceleration region is located below Juno or (ii) the plasma density is sometimes too dense for CMI instability to develop (and that $\mathrm{f}_{\mathrm{pe}} / \mathrm{f}_{\mathrm{ce}}>0.1$, as at Saturn).

Looking at the Juno/Jovian Auroral Distributions Experiment (McComas et al., 2017) electron data in the vicinity of source crossings will help us to (i) test the plasma density, as well as the presence of field aligned 


\section{Acknowledgments}

The authors thank the Juno mission team, especially the staff of the Waves instrument, and the APIS service at LESIA/Paris Astronomical Data Centre (Observatoire de Paris, CNRS) for providing processed UV observations (http://apis.obspm.fr/) acquired with the ESA/NASA Hubble Space Telescope (GO Program 14634). The Juno/Waves data used in this paper are reachable from the Planetary Data System (http://pds.nasa.gov/). This work was supported by the CNES and CNRS/INSU programs of Planetology (PNP) and Heliophysics (PNST). C. K. L. acknowledges funding support from the CNES, the Plas@par LABEX project, and the Agence Nationale de la Recherche (ANR-11-IDEX-0004-02). The research at the University of Iowa was supported by NASA through Contract 699041X with the Southwest Research Institute. currents and accelerated electron beams (which are the two necessary conditions for the CMI to exist), and (ii) better understand and constrain the reasons of the appearance/disappearance of radio emissions in the precipitation regions. Moreover, it will be interesting to compare each local radio source crossing with the many more FUV observations made by the Juno/UVS instrument (as done by ; Imai et al., 2019, for three PJs and bKOM sources) for all the perijoves. But this is beyond the scope of this study and may be the subject of a complementary study. The analysis of more bKOM, HOM, and DAM source crossings will make it possible to (i) refine the range of MFL hosting the sources and (ii) characterize the properties of these sources like the cutoff frequency and saturation process of these emissions. With the cutoff frequency, it will be possible to test the emission frequency of the CMI and the direction of propagation of these emissions (perpendicularly or obliquely to the MFLs).

\section{References}

Bagenal, F., Adriani, A., Allegrini, F., Bolton, S. J., Bonfond, B., Bunce, E. J., et al. (2017). Magnetospheric science objectives of the Juno mission. Space Science Reviews, 213, 219-287. https://doi.org/10.1007/s11214-014-0036-8

Clarke, J. T., Nichols, J., Gérard, J.-C., Grodent, D., Hansen, K. C., Kurth, W., et al. (2009). Response of Jupiter's and Saturn's auroral activity to the solar wind. Journal of Geophysical Research, 114, A05210. https://doi.org/10.1029/2008JA013694

Connerney, J. E. P., Acuna, M. H., \& Ness, N. F. (1981). Modeling the Jovian current sheet and inner magnetosphere. Journal of Geophysics Research, 86, 8370-8384. https://doi.org/10.1029/JA086iA10p08370

Connerney, J. E. P., Baron, R., Satoh, T., \& Owen, T. (1993). Images of excited $\mathrm{H}_{3}^{+}$at the foot of the Io flux tube in Jupiter's atmosphere. Science, 262, 1035-1038. https://doi.org/10.1126/science.262.5136.1035

Connerney, J. E. P., Benn, M., Bjarno, J. B., Denver, T., Espley, J., Jorgensen, J. L., et al. (2017). The Juno magnetic field investigation. Space Science Reviews, 213, 39-138. https://doi.org/10.1007/s11214-017-0334-z

Connerney, J. E. P., Kotsiaros, S., Oliversen, R. J., Espley, J. R., Joergensen, J. L., Joergensen, P. S., et al. (2018). A new model of Jupiter's magnetic field from Juno's first nine orbits. Geophysical Research Letters, 45, 2590-2596. https://doi.org/10.1002/2018GL077312

Cowley, S. W. H., Nichols, J. D., \& Bunce, E. J. (2002). Distributions of current and auroral precipitation in Jupiter's middle magnetosphere computed from steady-state Hill-Pontius angular velocity profiles: Solutions for current sheet and dipole magnetic field models. Planetary Space Science, 50, 717-734. https://doi.org/10.1016/S0032-0633(02)00046-6

Genova, F., Zarka, P., \& Barrow, C. H. (1987). Voyager and Nancay observations of the Jovian radio-emission at different frequencies-Solar wind effect and source extent. Astronomy \& Astrophysics, 182, 159-162.

Gladstone, G. R., Persyn, S. C., Eterno, J. S., Walther, B. C., Slater, D. C., Davis, M. W., et al. (2017). The ultraviolet spectrograph on NASA's Juno mission. Space Science Reviews, 213, 447-473. https://doi.org/10.1007/s11214-014-0040-z

Grodent, D. (2015). A brief review of ultraviolet auroral emissions on giant planets. Space Science Reviews, 187, 23-50. https://doi.org/10. 1007/s11214-014-0040-z

Grodent, D., Bonfond, B., Yao, Z., Gérard, J.-C., Radioti, A., Dumont, M., et al. (2018). Jupiter's aurora observed with HST during Juno Orbits 3 to 7. Journal of Geophysical Research: Space Physics, 123, 3299-3319. https://doi.org/10.1002/2017JA025046

Gurnett, D. A., Kurth, W. S., Hospodarsky, G. B., Persoon, A. M., Zarka, P., Lecacheux, A., et al. (2002). Control of Jupiter's radio emission and aurorae by the solar wind. Nature, 415, 985-987. https://doi.org/10.1038/415985a

Gustin, J., Grodent, D., Ray, L. C., Bonfond, B., Bunce, E. J., Nichols, J. D., \& Ozak, N. (2016). Characteristics of north Jovian aurora from STIS FUV spectral images. Icarus, 268, 215-241. https://doi.org/10.1016/j.icarus.2015.12.048

Hilgers, A. (1992). The auroral radiating plasma cavities. Geophysical Research Letters, 19, 237-240. https://doi.org/10.1029/91GL02938

Huff, R. L., Calvert, W., Craven, J. D., Frank, L. A., \& Gurnett, D. A. (1988). Mapping of auroral kilometric radiation sources to the aurora. Journal of Geophysics Research, 93, 11,445-11,454. https://doi.org/10.1029/JA093iA10p11445

Imai, M., Greathouse, T. K., Kurth, W. S., Randall, G., Louis, C. K., Zarka, P., et al. (2019). Probing Jovian broadband kilometric radio sources tied to the ultraviolet main auroral oval with Juno. Geophysical Research Letters, 46, 571-579. https://doi.org/10.1029/2018GL081227

Imai, M., Kurth, W. S., Hospodarsky, G. B., Bolton, S. J., Connerney, J. E. P., \& Levin, S. M. (2017). Direction-finding measurements of Jovian low-frequency radio components by Juno near Perijove 1. Geophysical Research Letters, 44, 6508-6516. https://doi.org/10.1002/ 2017GL072850

Kurth, W. S., Gurnett, D. A., Clarke, J. T., Zarka, P., Desch, M. D., Kaiser, M. L., et al. (2005). An Earth-like correspondence between Saturn's auroral features and radio emission. Nature, 433, 722-725. https://doi.org/10.1038/nature03334

Kurth, W. S., Hospodarsky, G. B., Kirchner, D. L., Mokrzycki, B. T., Averkamp, T. F., Robison, W. T., et al. (2017). The Juno Waves investigation. Space Science Reviews, 213, 347-392. https://doi.org/10.1007/s11214-017-0396-y

Kurth, W. S., Imai, M., Hospodarsky, G. B., Gurnett, D. A., Louarn, P., Valek, P., et al. (2017). A new view of Jupiter's auroral radio spectrum. Geophysical Research Letters, 44, 7114-7121. https://doi.org/10.1002/2017GL072889

Ladreiter, H. P., Zarka, P., \& Lecacheux, A. (1994). Direction finding study of Jovian hectometric and broadband kilometric radio emissions: Evidence for their auroral origin. Planetary Space Science, 42, 919-931. https://doi.org/10.1016/0032-0633(94)90052-3

Lamy, L., Cecconi, B., Prangé, R., Zarka, P., Nichols, J. D., \& Clarke, J. T. (2009). An auroral oval at the footprint of Saturn's kilometric radio sources, colocated with the UV aurorae. Journal of Geophysical Research, 114, A10212. https://doi.org/10.1029/2009JA014401

Lamy, L., Prangé, R., Henry, F., \& Le Sidaner, P. (2015). The Auroral Planetary Imaging and Spectroscopy (APIS) service. Astronomy and Computing, 11, 138-145. https://doi.org/10.1016/j.ascom.2015.01.005

Lamy, L., Prangé, R., Pryor, W., Gustin, J., Badman, S. V., Melin, H., et al. (2013). Multispectral simultaneous diagnosis of Saturn's aurorae throughout a planetary rotation. Journal of Geophysical Research: Space Physics, 118, 4817-4843. https://doi.org/10.1002/jgra.50404

Lamy, L., Zarka, P., Cecconi, B., Prangé, R., Kurth, W. S., Hospodarsky, G., et al. (2018). The low frequency source of Saturn's kilometric radiation. Science, 362, 6410. https://doi.org/10.1126/science.aat2027

Louarn, P., Allegrini, F., McComas, D. J., Valek, P. W., Kurth, W. S., André, N., et al. (2017). Generation of the Jovian hectometric radiation: First lessons from Juno. Geophysical Research Letters, 44, 4439-4446. https://doi.org/10.1002/2017GL072923

McComas, D. J., Alexander, N., Allegrini, F., Bagenal, F., Beebe, C., Clark, G., et al. (2017). The Jovian Auroral Distributions Experiment (JADE) on the Juno mission to Jupiter. Space Science Reviews, 213, 547-643. https://doi.org/10.1007/s11214-013-9990-9 
Menietti, J. D., Mutel, R. L., Christopher, I. W., Hutchinson, K. A., \& Sigwarth, J. B. (2011). Simultaneous radio and optical observations of auroral structures: Implications for AKR beaming. Journal of Geophysical Research, 116, A12219. https://doi.org/10.1029/2011JA017168

Nichols, J., \& Cowley, S. (2004). Magnetosphere-ionosphere coupling currents in Jupiter's middle magnetosphere: effect of precipitation-induced enhancement of the ionospheric Pedersen conductivity. Annales Geophysicae, 22, 1799-1827. https://doi.org/10. 5194/angeo-22-1799-2004

Pallier, L., \& Prangé, R. (2004). Detection of the southern counterpart of the Jovian northern polar cusp: Shared properties. Geophysical Research Letters, 31, L06701. https://doi.org/10.1029/2003GL018041

Prangé, R., Rego, D., Pallier, L., Connerney, J., Zarka, P., \& Queinnec, J. (1998). Detailed study of FUV Jovian auroral features with the post-COSTAR HST faint object camera. Journal of Geophysics Research, 103, 20,195-20,216. https://doi.org/10.1029/98JE01128

Prangé, R., Rego, D., Southwood, D., Zarka, P., Miller, S., \& Ip, W. (1996). Rapid energy dissipation and variability of the lo-Jupiter electrodynamic circuit. Nature, 379, 323-325. https://doi.org/10.1038/379323a0

Prangé, R., Zarka, P., Ballester, G. E., Livengood, T. A., Denis, L., Carr, T., et al. (1993). Correlated variations of UV and radio emissions during an outstanding Jovian auroral event. Journal of Geophysics Research, 98, 18. https://doi.org/10.1029/93JE01802

Reiner, M. J., Fainberg, J., \& Stone, R. G. (1993). Source characteristics and locations of hectometric radio emissions from the northern Jovian hemisphere. Geophysical Research Letters, 20, 321-324. https://doi.org/10.1029/93GL00245

Reiner, M. J., Fainberg, J., \& Stone, R. G. (1993). Source characteristics of Jovian hectometric radio emissions. Journal of Geophysical Research, 98, 18. https://doi.org/10.1029/93JE01779

Szalay, J. R., Bonfond, B., Allegrini, F., Bagenal, F., Bolton, S., Clark, G., et al. (2018). In situ observations connected to the Io footprint tail aurora. Journal of Geophysical Research: Planets, 123, 3061-3077. https://doi.org/10.1029/2018JE005752

Vincent, M. B., Clarke, J. T., Ballester, G. E., Harris, W. M., West, R. A., Trauger, J. T., et al. (2000). Mapping Jupiter's latitudinal bands and Great Red Spot using HST/WFPC2 far-ultraviolet imaging. Icarus, 143, 189-204. https://doi.org/10.1006/icar.1999.6232

Wu, C. S. (1985). Kinetic cyclotron and synchrotron maser instabilities-Radio emission processes by direct amplification of radiation. Space Science Reviews, 41, 215-298. https://doi.org/10.1007/BF00190653

Zarka, P. (1998). Auroral radio emissions at the outer planets: Observations and theories. Journal of Geophysics Research, 103, 20,159-20,194. https://doi.org/10.1029/98JE01323

Zarka, P. (2004). Radio and plasma waves at the outer planets. Advances in Space Research, 33, 2045-2060. https://doi.org/10.1016/j.asr. 2003.07.055

Zarka, P., Cecconi, B., \& Kurth, W. S. (2004). Jupiter's low-frequency radio spectrum from Cassini/Radio and Plasma Wave Science (RPWS) absolute flux density measurements. Journal of Geophysical Research, 109, A09S15. https://doi.org/10.1029/2003JA010260

Zarka, P., Queinnec, J., \& Crary, F. J. (2001). Low-frequency limit of Jovian radio emissions and implications on source locations and Io plasma wake. Planetary Space Science, 49, 1137-1149. https://doi.org/10.1016/S0032-0633(01)00021-6 